\title{
Lifespan method as a tool to study criticality in absorbing-state phase transitions
}

\author{
Angélica S. Mata, ${ }^{1,2}$ Marian Boguñá, ${ }^{3}$ Claudio Castellano,,${ }^{4,5}$ and Romualdo Pastor-Satorras ${ }^{2}$ \\ ${ }^{1}$ Departamento de Física, Universidade Federal de Viçosa, 36571-000 Viçosa, Minais Gerais, Brazil \\ ${ }^{2}$ Departament de Física i Enginyeria Nuclear, Universitat Politècnica de Catalunya, Campus Nord B4, 08034 Barcelona, Spain \\ ${ }^{3}$ Departament de Física Fonamental, Universitat de Barcelona, Martí i Franquès 1, 08028 Barcelona, Spain \\ ${ }^{4}$ Istituto dei Sistemi Complessi (ISC-CNR), via dei Taurini 19, I-00185 Roma, Italy \\ ${ }^{5}$ Dipartimento di Fisica, “Sapienza” Università di Roma, Piazzale Aldo Moro 2, I-00185 Roma, Italy
}

(Received 21 November 2014; published 12 May 2015)

\begin{abstract}
In a recent work, a new numerical method (the lifespan method) has been introduced to study the critical properties of epidemic processes on complex networks [M. Boguñá, C. Castellano, and R. Pastor-Satorras, Phys. Rev. Lett. 111, 068701 (2013)]. Here, we present a detailed analysis of the viability of this method for the study of the critical properties of generic absorbing-state phase transitions in lattices. Focusing on the well-understood case of the contact process, we develop a finite-size scaling theory to measure the critical point and its associated critical exponents. We show the validity of the method by studying numerically the contact process on a one-dimensional lattice and comparing the findings of the lifespan method with the standard quasistationary method. We find that the lifespan method gives results that are perfectly compatible with those of quasistationary simulations and with analytical results. Our observations confirm that the lifespan method is a fully legitimate tool for the study of the critical properties of absorbing phase transitions in regular lattices.
\end{abstract}

DOI: 10.1103/PhysRevE.91.052117

PACS number(s): 05.70.Jk, 05.10.Gg, 64.60.an

\section{INTRODUCTION}

A key class of dynamical nonequilibrium systems includes those with absorbing states, i.e., states from which the dynamics cannot escape once it falls on them. Classical examples of such systems are epidemic spreading processes [1]; obviously, a fully healthy state is absorbing in the above sense, provided we do not allow for immigration of infected individuals. A very relevant feature of many systems with absorbing states is their ability to exhibit absorbing-state phase transitions [2,3], that is, nonequilibrium phase transitions between an active state, characterized by everlasting activity in the thermodynamic limit, and an absorbing state, where activity is absent.

Apart from a few exactly solvable models [4], the theoretical characterization of absorbing-state phase transitions is based usually on mean-field theories [3], field theory renormalization procedures [5], topological phase-space field theories [6], dynamical mean-field plus coherent anomaly extrapolation [7], or series expansions for particular models [8,9]. While simple mean-field theory is only valid above the upper critical dimension, application of other techniques in physical dimensions is usually hindered by technical difficulties. For this reason, a large amount of our knowledge about the properties of absorbing-state phase transitions is based on computer simulation of different representative models. The numerical analysis of these computer data represents a different sort of challenge, which is mainly hampered by finite size effects. In finite systems, any realization of the dynamics is bound to reach sooner or later the absorbing state, even in the active phase, due to dynamic fluctuations. This difficulty can be overcome by applying the finite-size scaling technique [10], based on the size dependence of physical observables that are averaged only over surviving runs, i.e., realizations which have not yet fallen into the absorbing state [3]. The critical point and various critical exponents can then be determined by studying the decay of the average of different observables over surviving runs as a function of the system size. Averaging over surviving runs is, however, computationally highly inefficient. A more effective alternative is provided by the quasistationary (QS) method [11-13], in which every time the system tries to visit an absorbing state, it jumps instead to an active configuration previously stored during the simulation.

Recently, in the context of epidemic modeling on complex networks [14], Boguñá et al. [15], building on the traditional method of seed simulations [2], proposed to consider the lifespan of spreading simulations starting from a single infected site as a tool to determine the position of the critical point. Inspired by the satisfactory performance of the lifespan (LS) method on epidemic models in networks [15], in this paper we consider its extension and application to models with absorbing states on regular Euclidean lattices, presenting a detailed finite-size scaling theory for this approach to determine critical properties of absorbing-state phase transitions. To provide a concrete application example, we focus on the well-known controlled case of the contact process (CP) [16] in a one-dimensional lattice, for which theoretical and high-quality numerical results are already available. In this way, we are able to make a direct assessment of the reliability of the LS method. A close comparison with the results of quasistationary simulations is also performed. Our results indicate that the lifespan method is a perfectly viable alternative to investigate critical properties of absorbing phase transitions in regular lattices.

We have organized our paper as follows: In Sec. II we define the $\mathrm{CP}$ and present the numerical implementation and main properties of this model. Section III reviews briefly the QS method and the finite-size scaling form of the properties computed from it. Sections IV and V present the LS method and discuss its finite-size scaling theory, respectively. In Sec. VI, we present numerical results comparing the predictions of both QS and LS methods for the CP in a $d=1$ lattice. Conclusions and perspectives are finally discussed in Sec. VII. 


\section{THE CONTACT PROCESS}

The CP represents the simplest theoretical model with an absorbing-state phase transition [16]. The CP is defined as follows: Sites in a lattice are characterized by a binary variable $\sigma_{i}$ that can take values $\sigma_{i}=1$ (occupied by a particle) or $\sigma_{i}=$ 0 (empty). Each occupied vertex can spontaneously become empty at a rate which, without loss of generality, is set equal to 1 , thus fixing the time scale. On the other hand, at a rate $\lambda / z$, where $z$ is the coordination number of the lattice, an occupied site creates offspring particles on its empty nearest neighbors (note that all temporal processes are assumed to be Poisson point processes). The creation of particles is a catalytic process occurring exclusively in pairs of empty-occupied sites, implying that the state devoid of particles is a fixed point of the dynamics (i.e., an absorbing state).

On a lattice with $N$ nodes, the $\mathrm{CP}$ is numerically simulated as follows [3]: An occupied site $j$ is randomly selected. With probability $p=1 /(1+\lambda)$ the selected site becomes empty. With complementary probability $1-p$ one of the neighbors of $j$ is randomly chosen and, if empty, it becomes occupied. Time is incremented by $\Delta t=1 /[(1+\lambda) n(t)]$, where $n(t)$ is the number of occupied sites at time $t$. We note that this prescription [3] (consistent with a variation of the classical Gillespie algorithm [17,18] in which time is incremented in a deterministic way) corresponds to a sequential update of events. This is the only way to reproduce offspring creation events among occupied and empty sites taking place at a rate $\lambda / z$ according to a Poisson point process.

In an infinite system, the $\mathrm{CP}$ displays an absorbing-state phase transition at a critical point $\lambda_{c}$, between an absorbing phase for $\lambda \leqslant \lambda_{c}$, and an active one for $\lambda>\lambda_{c}$. The order parameter of the transition is the stationary density of occupied sites $\rho_{\mathrm{st}}(\lambda) \equiv \lim _{t \rightarrow \infty} \lim _{N \rightarrow \infty}\langle n(t)\rangle / N$, which is zero below the threshold $\lambda_{c}$ and larger than zero above it. Near the critical point $\rho_{\mathrm{st}}(\lambda)$ vanishes as a power law

$$
\rho_{\mathrm{st}}(\lambda) \propto\left(\lambda-\lambda_{c}\right)^{\beta},
$$

characterized by the critical exponent $\beta$. The onset of critical fluctuations at the transition is ruled by a diverging correlation length $\xi$, given by

$$
\xi \propto\left|\lambda-\lambda_{c}\right|^{-v_{\perp}},
$$

where $v_{\perp}$ is the finite-size scaling exponent.

\section{THE QUASISTATIONARY METHOD}

The standard numerical procedure to investigate the finitesize scaling at absorbing phase transitions-by measuring the average of the order parameter restricted only to surviving runs-is extremely inefficient, since surviving configurations are very rare at long times. The quasistationary method represents an alternative strategy which consists in constraining the system to be in a quasistationary state. In practice, this is implemented by replacing the absorbing state, every time the system tries to visit it, with an active configuration randomly taken from the history of the simulation [11]. For this task, a list of $M$ active configurations is stored and constantly updated. An update consists in randomly choosing a configuration in the list and replacing it by the present active configuration with a small probability $p_{r} \Delta t \ll 1$. The parameter $p_{r}$ is typically chosen to be equal to 0.02 . In any case, in the simulations presented here, no significant dependence on this parameter was detected for a wide range of variations in simulations.

After a relaxation time, the QS quantities are determined during a given averaging time window. Following this approach, it is possible to evaluate the full probability distribution of the number of occupied vertices in the quasistationary state and use it to calculate all quantities of interest. The transition point is then determined by considering the modified susceptibility [19]

$$
\chi=\frac{L^{d}\left(\left\langle\rho^{2}\right\rangle-\langle\rho\rangle^{2}\right)}{\langle\rho\rangle} .
$$

Close to the critical point, the susceptibility diverges as $\chi \sim\left(\lambda_{c}-\lambda\right)^{-(\gamma+\beta)}$. As we see, the critical exponent of this susceptibility is larger than the standard one $(\gamma)$, which simplifies its numerical evaluation while preserving all the scaling properties. In a finite lattice of side $L, \chi$ shows a diverging peak at $\lambda=\lambda_{p}^{\mathrm{QS}}(L)$, providing a finite size approximation of the critical point. In the thermodynamic limit, $\lambda_{p}^{\mathrm{QS}}(L)$ approaches the true critical point with the scaling form [20]

$$
\lambda_{p}^{\mathrm{QS}}(L)=\lambda_{c}+A_{\mathrm{QS}} L^{-1 / \nu_{\perp}} .
$$

In finite but large systems, the density of occupied sites and the susceptibility can be written near the critical point with the finite-size scaling form ${ }^{1}$ [3]

$$
\rho_{\mathrm{st}}(\lambda, L) \propto L^{-\beta / v_{\perp}} f\left[\left(\lambda-\lambda_{c}\right) L^{1 / \nu_{\perp}}\right],
$$

and

$$
\chi(\lambda, L) \propto L^{(\gamma+\beta) / \nu_{\perp}} g\left[\left(\lambda-\lambda_{c}\right) L^{1 / \nu_{\perp}}\right],
$$

where $f(x)$ and $g(x)$ are scaling functions that satisfy $f(x) \propto$ $x^{\beta}$ for $x \gg 1, f(x) \propto|x|^{-v_{\perp}+\beta}$ for $-x \gg 1$, and $f(x)=\mathrm{const}$ for $|x| \ll 1$, and $g(x) \propto|x|^{-(\gamma+\beta)}$ for $|x| \gg 1, g(x)=\mathrm{const}$ for $|x| \ll 1$. Equations (5) and (6) imply that, at the critical point, the QS observables depend on $L$ as

$$
\rho_{\mathrm{st}}\left(\lambda_{c}, L\right) \propto L^{-\beta / \nu_{\perp}} \quad \text { and } \quad \chi\left(\lambda_{c}, L\right) \propto L^{(\gamma+\beta) / \nu_{\perp}} .
$$

\section{THE LIFESPAN METHOD}

The LS method proposed by Boguñá et al. [15] considers spreading simulations starting from a single occupied site. Each realization of the dynamical process is characterized by its lifespan $\tau$ and its coverage $C$, where the latter is defined as the number of distinct sites which have been occupied at least once during the realization. In the thermodynamic limit, realizations can be either finite or endemic. Endemic realizations have an infinite lifespan and their coverage is equal to the system size; such realizations are only possible above the critical point. Finite realizations, on the other hand, have finite lifespan and coverage. Finite realizations can be found both below and above the critical point, although the probability to

\footnotetext{
${ }^{1}$ Here and in the following we do not consider the possibility of the breakdown of standard finite-size scaling forms due to dangerously irrelevant scaling fields [21].
} 
find a finite realization decreases when $\lambda$ is increased above the critical point.

In the LS method, the role of the order parameter is played by the probability that a run is endemic, $\mathrm{P}_{\mathrm{end}}(\lambda)$. This probability is zero below the critical point and grows monotonously for $\lambda>\lambda_{c}$, approaching 1 in the limit $\lambda \rightarrow \infty$. The role of the susceptibility is played by the average lifetime of finite realizations $\langle\tau\rangle$. For small values of $\lambda$ all realizations are finite and have a very short duration. As $\lambda$ grows the average duration of finite realizations increases, diverging at the critical point. Above the critical point, the probability of a realization to be endemic increases and those realizations that remain finite have necessarily a short lifespan. This is so because once a realization has been alive for a very long time, the probability that it becomes finally endemic increases. As a result, $\langle\tau\rangle$ diverges when approaching the critical point from the left and decreases as $\lambda$ is increased further. In a finite system with $N$ nodes, $\langle\tau\rangle$ exhibits a peak for a value $\lambda_{p}^{\mathrm{LS}}(N)$ that converges to $\lambda_{c}$ in the thermodynamic limit.

In finite systems, the program described above has to be implemented with care. Indeed, in a finite system any realization is bound to end, reaching the absorbing state, even though this might occur over astronomically long temporal scales. Therefore, the distinction between finite and endemic realizations is, a priori, not clear cut. In practice, we declare a realization as endemic whenever its coverage fraction reaches a predefined threshold value $C_{\text {th }}=c_{\text {th }} N$, with $c_{\text {th }}$ a constant value between zero and one. Realizations ending before the value $C=C_{\text {th }}$ is reached are considered to be finite. In the thermodynamic limit, reaching $C_{\mathrm{th}}$ means that an infinite number of nodes have been reached by the outbreak. If so, the probability that such a realization is eventually trapped in the absorbing state is zero, meaning that the realization is endemic with probability 1 .

\section{FINITE-SIZE SCALING OF THE LIFESPAN METHOD}

In this section, we present a finite-size scaling theory of the LS method, which enables the detailed analysis of numerical simulations. In general, the theory can be applied to any type of discrete structure. For this reason, hereafter we use the number of sites, $N$, as the measure of the size of the system. The case of a lattice of side $L$ in $d$ dimensions can be easily recovered by replacing $N=L^{d}$. Let $\Psi(\tau, C ; \lambda)$ be the joint probability of a realization of the $\mathrm{CP}$ process to have, in an infinite size system, a (finite) lifespan $\tau$ and coverage $C$. This joint probability can be written as

$$
\Psi(\tau, C ; \lambda)=\psi(\tau ; \lambda) \Theta(C \mid \tau ; \lambda),
$$

where $\psi(\tau ; \lambda)$ is the probability density of the lifespan $\tau$ and $\Theta(C \mid \tau ; \lambda)$ is the probability that the coverage is $C$, given that the lifespan is $\tau$. The usual scaling assumption for $\psi(\tau ; \lambda)$, near the critical point, is [3]

$$
\psi(\tau ; \lambda)=\tau^{-1-\delta} \hat{f}\left[\left(\lambda_{c}-\lambda\right) \tau^{\sigma}\right],
$$

for $\tau>\tau_{\min }$, some minimum time scale. The scaling function $\hat{f}(x)$ is nonsymmetric, is continuous at $x=0$, is constant when $|x| \ll 1$, and decays faster than a power law when $|x| \gg$ 1. The scaling hypothesis Eq. (9) can be used to derive a scaling relation between the exponent $\beta$ and the exponents $\delta$ and $\sigma$. Below the critical point, $\lambda<\lambda_{c}$, all realizations are finite and, thus, $\int \psi(\tau ; \lambda) d \tau=1$. Above this point, there is a finite probability that a realization is endemic and, therefore, $\int \psi(\tau ; \lambda) d \tau=1-\mathrm{P}_{\text {end }}(\lambda)$. Combining these two results and Eq. (9) leads to

$\mathrm{P}_{\mathrm{end}}(\lambda) \sim \frac{1}{\sigma}\left(\lambda-\lambda_{c}\right)^{\frac{\delta}{\sigma}} \int_{0}^{\infty} x^{-1-\frac{\delta}{\sigma}}[\hat{f}(x)-\hat{f}(-x)] d x$,

which provides the relation $\beta=\delta / \sigma$.

The scaling assumption Eq. (9) tells us that the lifespan is power law distributed up to the cutoff value

$$
\tau_{\text {cut }} \sim\left|\lambda_{c}-\lambda\right|^{-1 / \sigma},
$$

depending on the deviation from the critical point. ${ }^{2}$ In turn, this implies that, close to the critical point, the moments $\left\langle\tau^{n}\right\rangle$ behave as ${ }^{3}$

$$
\left\langle\tau^{n}\right\rangle \approx \int_{0}^{\tau_{\mathrm{cut}}} \tau^{n-1-\delta} d \tau \sim\left|\lambda_{c}-\lambda\right|^{\frac{\delta-n}{\sigma}} .
$$

This result is similar to the behavior of the size of finite clusters in regular percolation [22].

In finite systems, an additional temporal cutoff competes with $\tau_{\text {cut }}$ in Eq. (11), namely, the temporal cutoff $\bar{\tau}_{\text {cut }}(N)$ arising from the finiteness of the system size. To define this temporal scale, we consider the behavior near the critical point of the average coverage $\bar{C}\left(\tau ; \lambda_{c}\right) \equiv \sum_{C} C \Theta\left(C \mid \tau ; \lambda_{c}\right)$. At the critical point, we expect all physical observables to satisfy scaling relations. Thus, we can write

$$
\bar{C}\left(\tau ; \lambda_{c}\right) \sim \tau^{\mu} .
$$

However, since $\bar{C}\left(\tau ; \lambda_{c}\right)$ cannot become larger than $N$, Eq. (13) can only hold up to a cutoff value $\bar{\tau}_{\text {cut }}(N) \sim N^{1 / \mu}$. The interplay between the two cutoffs present in the system, $\tau_{\text {cut }} \sim\left|\lambda_{c}-\lambda\right|^{-1 / \sigma}$ (due to the distance from the critical point) and $\bar{\tau}_{\text {cut }}(N)$ (due to the finite size) determines the scaling of the moments $\left\langle\tau^{n}\right\rangle$. When $\bar{\tau}_{\text {cut }}(N) \gg \tau_{\text {cut }}$, the system does not notice its finiteness and, therefore, all moments are given by Eq. (12). Instead, when $\bar{\tau}_{\text {cut }}(N) \ll \tau_{\text {cut }}$, the distribution is cut off by $\bar{\tau}_{\text {cut }}(N)$ and, thus, all moments behave as $\left\langle\tau^{n}\right\rangle \sim \int^{\bar{\tau}_{\text {cut }}(N)} \tau^{n-1-\delta} d \tau \sim\left[\bar{\tau}_{\text {cut }}(N)\right]^{n-\delta}$. To sum up:

$$
\left\langle\tau^{n}\right\rangle \sim \begin{cases}\left|\lambda_{c}-\lambda\right|^{\frac{\delta-n}{\sigma}}, & \text { if }\left|\lambda_{c}-\lambda\right| N^{\sigma / \mu} \gg 1 \\ N^{\frac{n-\delta}{\mu}}, & \text { if }\left|\lambda_{c}-\lambda\right| N^{\sigma / \mu} \ll 1 .\end{cases}
$$

\footnotetext{
${ }^{2}$ Note that the prefactor in Eq. (11) can be different when approaching the critical point from below or from above.

${ }^{3}$ A more precise calculation using the scaling assumption Eq. (9) is given by

$$
\left\langle\tau^{n}\right\rangle=\frac{a_{ \pm}}{\sigma}\left|\lambda_{c}-\lambda\right|^{\frac{\delta-n}{\sigma}} \quad \text { with } \quad a_{ \pm}=\int_{0}^{\infty} x^{\frac{n-\delta}{\sigma}-1} f(\mp x) d x
$$

where the positive (negative) value means approaching the critical point from the right (left).

${ }^{4}$ Notice that the fluctuations of the coverage near its maximum value $C \lesssim N$ vanish and, thus, in this region Eq. (13) can be considered a deterministic equivalence between coverage and lifespan.
} 
Defining the exponents $\gamma_{n} \equiv(n-\delta) / \sigma$ and $\nu_{\perp} \equiv \mu / \sigma$, the behavior of Eq. (14) can be captured by the following finitesize scaling form:

$$
\left\langle\tau^{n}(N)\right\rangle=N^{\gamma_{n} / \nu_{\perp}} G_{n}\left[\left(\lambda_{c}-\lambda\right) N^{1 / \nu_{\perp}}\right],
$$

where the scaling function $G_{n}(x)$ is constant if $|x| \ll 1$ and goes as $|x|^{-\gamma_{n}}$ when $|x| \gg 1$. As usual, we expect to find a maximum of $\left\langle\tau^{n}(N)\right\rangle$ around a value $\lambda_{p}^{\mathrm{LS}}(N)$, which depends on the system size as

$$
\lambda_{p}^{\mathrm{LS}}(N)=\lambda_{c}+A_{\mathrm{LS}} N^{-1 / v_{\perp}} .
$$

We can then use, in general, the average lifespan to determine numerically the critical point and some of the critical exponents. There is, however, a pathological case if the exponent of the lifespan distribution is exactly $\delta=1$. In such a case, the average lifespan $\langle\tau\rangle$ does diverge, but logarithmically; the critical point can still be determined but critical exponents cannot. This problem disappears if one uses the second moment $\left\langle\tau^{2}\right\rangle$ instead.

Finally, concerning the order parameter $\mathrm{P}_{\text {end }}(\lambda, N)$, defined as the probability that a run is endemic, it fulfills the standard finite-size scaling form

$$
\mathrm{P}_{\mathrm{end}}(\lambda, N) \propto N^{-\beta / \nu_{\perp}} f\left[\left(\lambda-\lambda_{c}\right) N^{1 / \nu_{\perp}}\right] .
$$

From this expression, we can determine the exponent $\beta / \nu_{\perp}$ by examining the $N$ dependence of $\mathrm{P}_{\text {end }}(\lambda, N)$ at the critical point:

$$
\mathrm{P}_{\text {end }}\left(\lambda_{c}, N\right) \propto N^{-\beta / \nu_{\perp}} .
$$

The missing piece of the scaling theory presented above is the value of the exponent $\mu$, governing the scaling with the system size of the lifespan cutoff at criticality $\bar{\tau}_{\text {cut }}(N)$. As we check numerically below, this cutoff can be identified in regular lattices with the characteristic relaxation time, which close to criticality scales as $\bar{\tau} \sim\left|\lambda-\lambda_{c}\right|^{-\nu_{\|}}$[3]. Comparing this relation with Eq. (11) leads to the identity $\mu=v_{\perp} / \nu_{\|}$.

\section{NUMERICAL RESULTS}

The critical properties of the $\mathrm{CP}$ on a one-dimensional lattice and the corresponding finite-size scaling theory for the transition are very well known, and accurate theoretical and numerical values are readily available for comparison [3]. This makes the $\mathrm{CP}$ on a one-dimensional lattice the ideal test bed for numerical methods. In this section, we present results of numerical simulations of the $\mathrm{CP}$ on a $d=1$ lattice, applying both the QS and LS methods. Hereafter, we use $N=L$.

\section{A. Quasistationary simulations}

In Fig. 1, we show the results obtained by performing QS simulations of the CP on a one-dimensional lattice of length $L$. The susceptibility $\chi$, Fig. 1(a), shows a well-defined peak, which becomes narrower and taller as the system size $L$ grows. The plot of the quasistationary density $\rho_{\mathrm{st}}(L)$, Fig. 1(b), also displays a transition becoming narrower and sharper as $L$ grows. From the position of the susceptibility peak $\lambda_{p}^{\mathrm{QS}}(N)$ it is possible to obtain asymptotically an estimate of the transition point $\lambda_{c}$ by applying the relation in Eq. (4). We have used this expression to perform a nonlinear regression to determine the
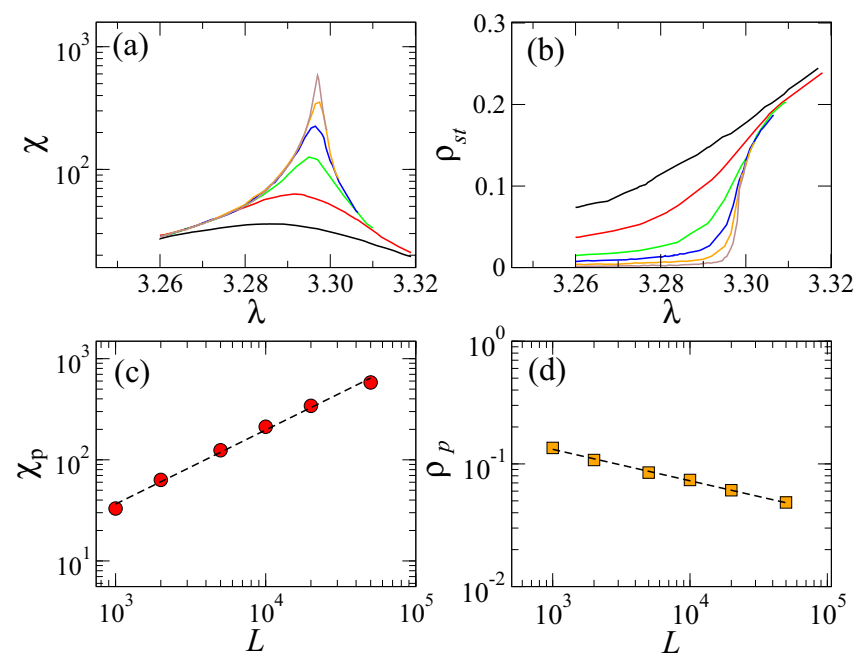

FIG. 1. (Color online) (a) Susceptibility and (b) density of active nodes as a function of $\lambda$ for quasistationary simulations of the $\mathrm{CP}$ on one-dimensional lattices of different sizes. System size is $L=1000$, 2000, 5000, 10000,20000 , and 50000 bottom to top in (a) and top to bottom in (b). Bottom plots show (c) the size dependence of the height of the peak of the susceptibility, $\chi_{p}(L)$, and (d) the quasistationary density evaluated at the peak of the susceptibility, $\rho_{p}(L)$. Dashed lines are power law fits of exponents $\beta / \nu_{\perp}=0.253(5)$ and $(\gamma+\beta) / \nu_{\perp}=$ $0.736(3)$. We performed 200 individual realizations and $t=2 \times 10^{6}$ Monte Carlo time steps. Error bars for $\chi_{p}(L)$ and $\rho_{p}(L)$ are smaller than symbols.

critical point $\lambda_{c}$ and the exponent $\nu_{\perp}$; see Fig. 2 and Table I. The values obtained by this procedure are in very good agreement with the best estimates accepted in the literature [2].

Right at the critical point, the average density of particles and the susceptibility should scale with the system size as given by Eq. (7). From this analysis [see Figs. 1(c) and 1(d)],

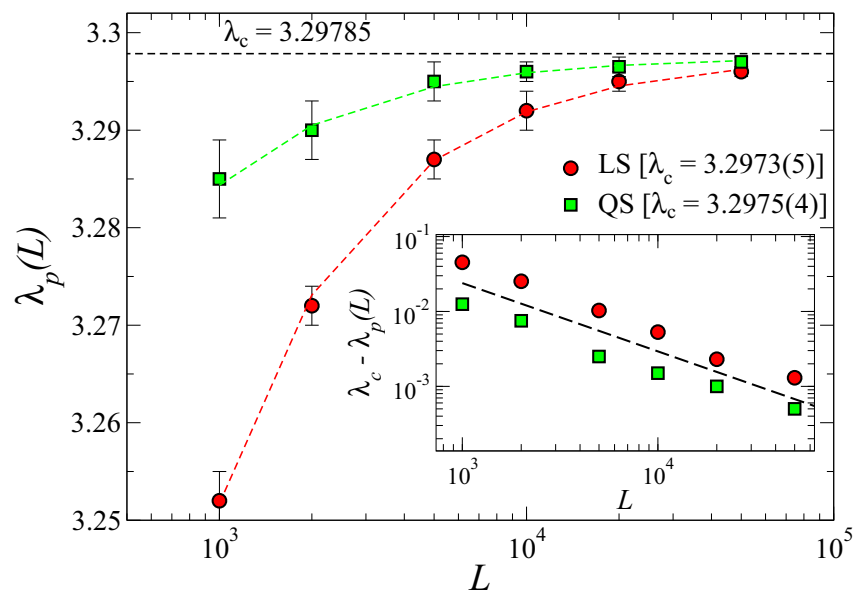

FIG. 2. (Color online) Size dependence of the $\lambda_{p}(L)$ estimates of the transition point for the quasistationary and lifespan methods. Dashed lines are nonlinear regressions used to determine the critical point and to estimate the critical exponent $v_{\perp}$, applying Eq. (4); see Table I. The horizontal line marks the accepted best estimate value of the critical point. The inset shows $\lambda_{c}-\lambda_{p}$ as a function of $L$ in $\log$-log scale. The dashed line has slope $1 / \nu_{\perp}$ as a guide to the eye. 
TABLE I. Critical point and exponents of the $\mathrm{CP}$ in a $d=1$ lattice obtained using the QS and LS methods. For comparison, we quote also the best estimates of those, from Ref. [2].

\begin{tabular}{llll}
\hline \hline & Theoretical & \multicolumn{1}{c}{ QS } & \multicolumn{1}{c}{ LS } \\
\hline$\lambda_{c}$ & $3.297848(22)$ & $3.2975(4)$ & $3.2973(5)$ \\
$\nu_{\perp}$ & $1.096854(4)$ & $1.098(5)$ & $1.100(5)$ \\
$\beta / \nu_{\perp}$ & $0.252068(8)$ & $0.253(5)$ & $0.255(5)$ \\
$(\gamma+\beta) / \nu_{\perp}$ & $0.74792(2)$ & $0.736(3)$ & \\
$\mu\left(=v_{\perp} / \nu_{\|}\right)$ & $0.632613(4)$ & & $0.64(1)$ \\
\hline \hline
\end{tabular}

we can compute the exponents $\beta / \nu_{\perp}$ and $(\gamma+\beta) / \nu_{\perp}$, which again reproduce with good accuracy the known values of the CP; see Table I.

\section{B. The lifespan method}

As discussed in Sec. V, in the LS method for finite systems, the role of the order parameter is played by the probability $\mathrm{P}_{\text {end }}(\lambda, L)$ that a run reaches the predefined coverage $C_{\text {th }}$ (i.e., it is effectively endemic), while the analog of the susceptibility is given by the average duration $\langle\tau\rangle$ of finite realizations.

In Fig. 3(a), we plot the average lifespan $\langle\tau\rangle$ as a function of $\lambda$, for different system sizes, computed for a fixed coverage
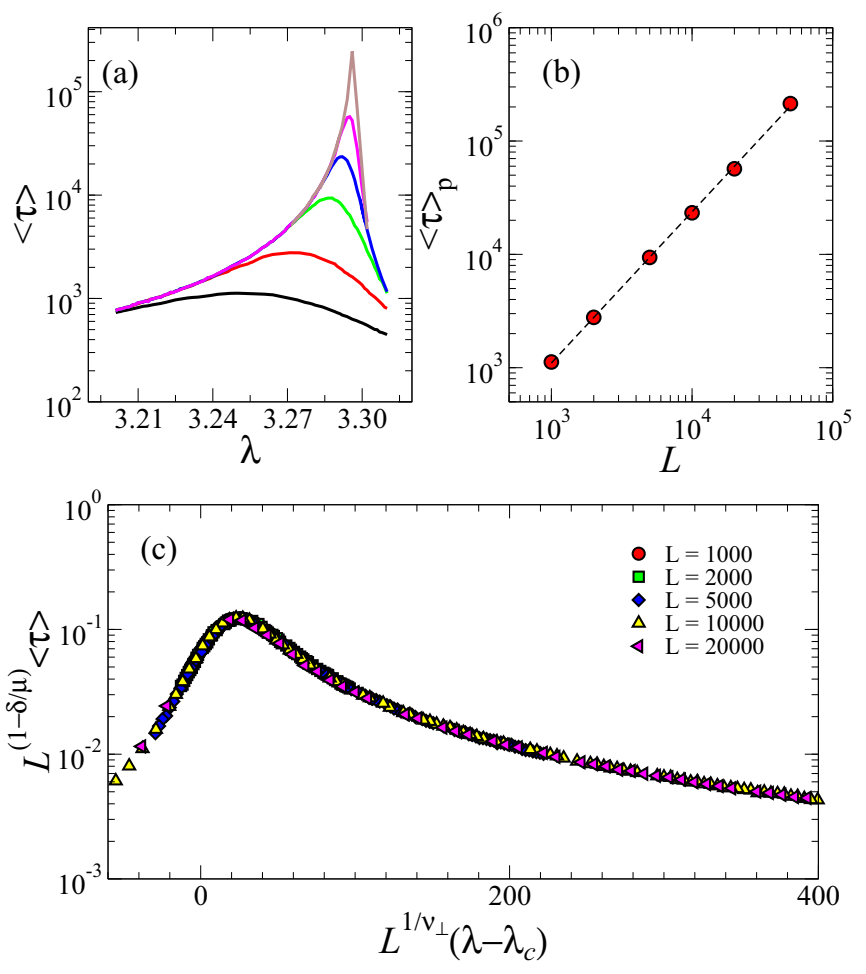

FIG. 3. (Color online) (a) Lifetime $\langle\tau\rangle$ against creation rate for the $\mathrm{CP}$ on a one-dimensional lattice. Curves are for system size (bottom to top) $L=1000,2000,5000,10000,20000$, and 50000. (b) Size dependence of the height of the peak of the average lifespan $\tau_{p}(L)$. The dashed line represents a power law regression with slope $(1-\delta) / \mu=1.32(1)$. We perform $5 \times 10^{5}$ individual realizations for each size. Error bars are smaller than symbols. (c) The scaling plot of the lifetime according to Eq. (15) for the same data of panel (a).

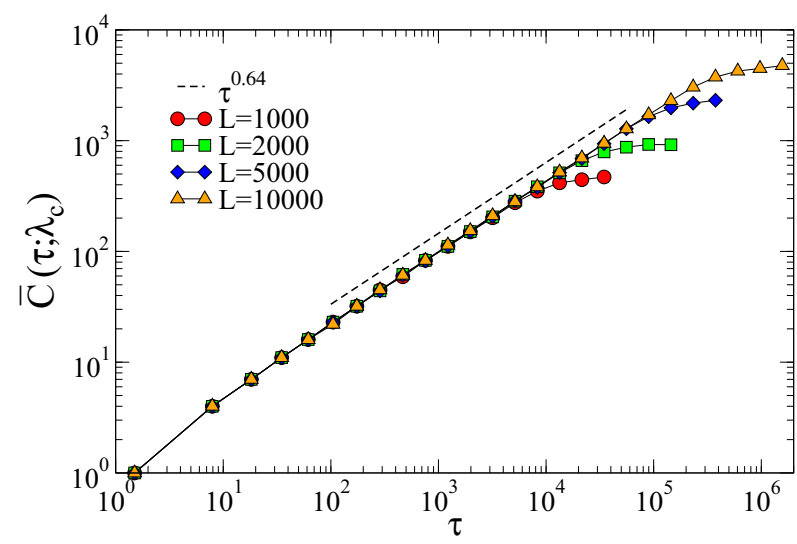

FIG. 4. (Color online) Average coverage as a function of $\tau$ evaluated at $\lambda=\lambda_{p}^{\mathrm{LS}}(N)$. The dashed line has slope 0.64 and serves as a guide to the eye.

fraction threshold $c_{\mathrm{th}}=0.5$; the effect of varying the coverage fraction threshold is discussed in Sec. VIC. From this figure, we can observe that the lifespan $\langle\tau\rangle$ has a well-defined peak at a value $\lambda_{p}^{\mathrm{LS}}(L)$, signaling the presence of a phase transition. The dependence of the peak position as a function of the system size $L$ is reported in Fig. 2. A nonlinear fitting of the data according to Eq. (4) provides numerical estimates for the critical point $\lambda_{c}$ and the exponent $\nu_{\perp}$ (see Table I), which are compatible with the exact results derived analytically. Hence, we conclude that both the QS and the LS methods recover compatible results for the position of the critical point and the exponent $v_{\perp}$.

The peak value $\langle\tau\rangle_{p}$ of the average lifespan grows as a power law as a function of $L$; see Fig. 3(b). According to the scaling theory presented in Sec. V, the exponent of this growth is equal to $(1-\delta) / \mu$, for which we obtain a value $1.32(1)$. The value of $\delta$ is well known in the literature, namely $\delta=0.159464(6)$ [2]. From here, we obtain the exponent $\mu=0.64(1)$. We can also determine this exponent directly from the scaling of the average coverage near the critical point, $\bar{C}\left(\tau ; \lambda_{c}\right) \sim \tau^{\mu}$; see Eq. (13). In Fig. 4, we analyze this coverage, obtaining numerically an exponent $\mu=0.64(1)$, in perfect agreement with the value found from the scaling of the peak of the average lifespan. In Fig. 3(c), we finally check the full finite-size scaling form of the lifespan $\langle\tau\rangle$ as given by Eq. (5). We perform a data collapse analysis by plotting $L^{(1-\delta) / \mu}\langle\tau\rangle$ as a function of $L^{1 / \nu_{\perp}}\left(\lambda-\lambda_{c}\right)$. The perfect collapse of the plots shown in Fig. 3(c) confirms the validity of the finite-size scaling proposed in Eq. (5).

Concerning the order parameter, in Fig. 5(a) we plot $\mathrm{P}_{\text {end }}(\lambda, L)$ evaluated with threshold coverage fraction $c_{\text {th }}=0.5$ as a function of $\lambda$ and different values of $L$. As we can see, it displays a sharp phase transition at the critical point when the size of the system increases. Close to criticality, and for large $L$, this probability exhibits a power law form with system size given by Eq. (18). By analyzing $\mathrm{P}_{\mathrm{end}}\left(\lambda_{c}, L\right)$ as a function of $L$, we can obtain the exponent $\beta / \nu_{\perp}$ (see Table I), again in very good agreement with QS estimates. Finally, in Fig. 5(b), we check the full finite-size scaling form Eq. (17) by plotting $L^{\beta / \nu_{\perp}} \mathrm{P}_{\text {end }}(\lambda, L)$ as a function of $L^{1 / \nu_{\perp}}\left(\lambda-\lambda_{c}\right)$, using the numerical exponents found. The perfect data collapse found 

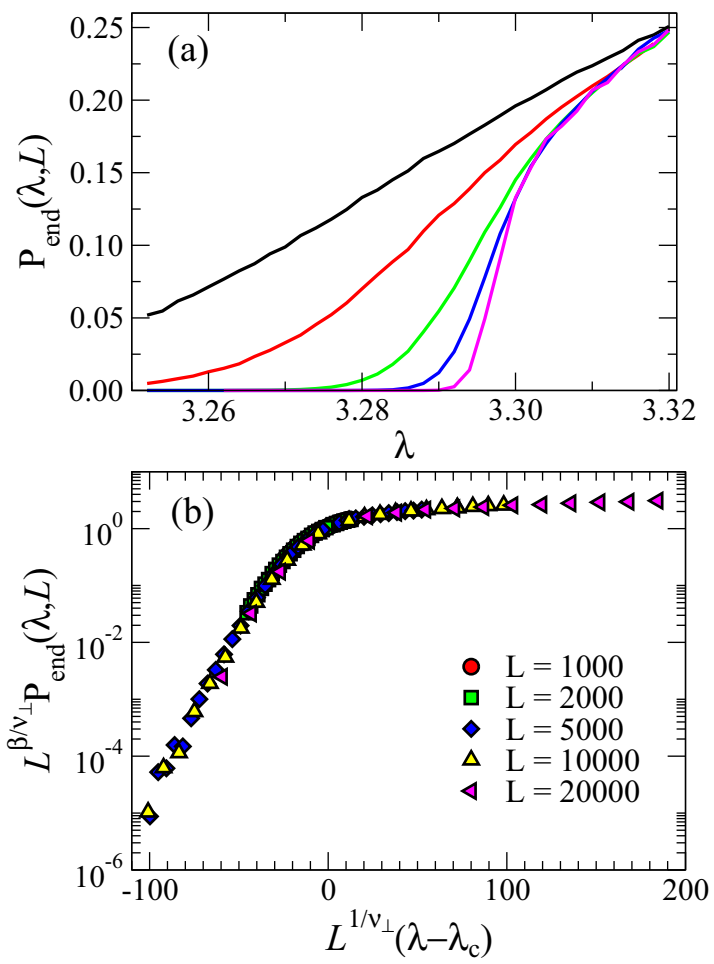

FIG. 5. (Color online) (a) Probability to reach the predefined coverage fraction $c_{\text {th }}=0.5$ as a function of $\lambda$ for the $\mathrm{CP}$ on a one-dimensional lattice. Curves are for system size (top to bottom) $L=1000,2000,5000,10000$, and 20 000. (b) Scaling plot of this probability according to Eq. (17).
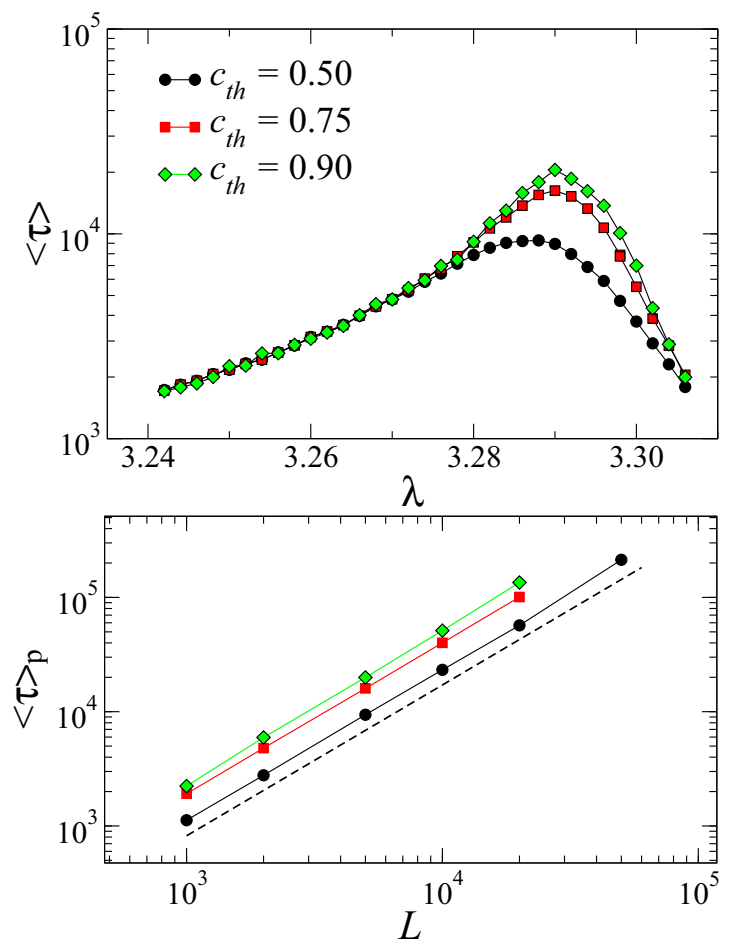

FIG. 6. (Color online) (a) The average lifespan $\langle\tau\rangle$ against creation rate for different values of $c_{\mathrm{th}}$, for $L=5000$. (b) The height of the peak grows with an exponent independent of $c_{\mathrm{th}}$. The dashed line has slope 1.32 .

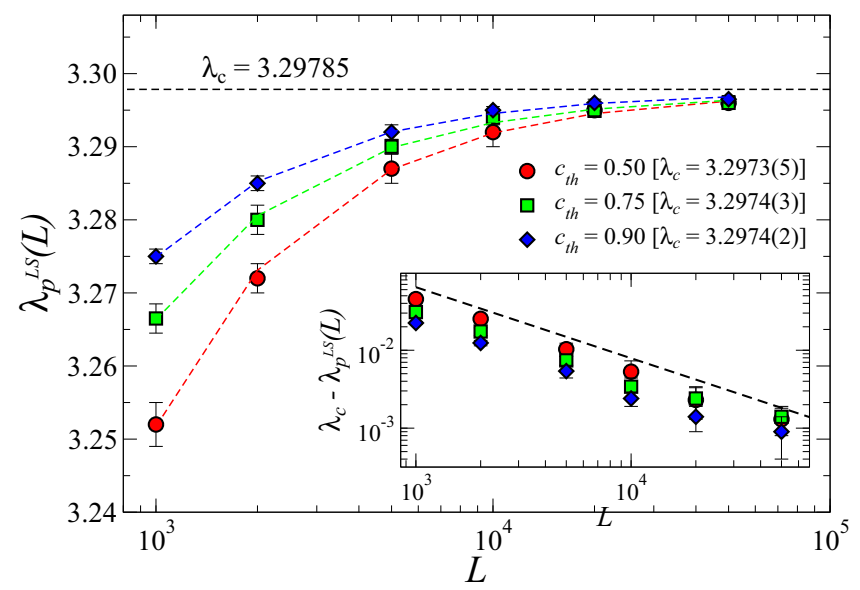

FIG. 7. (Color online) Size dependence of $\lambda_{p}(L)$ estimates with lifespan simulation method with different values of $c_{\text {th }}$. Inset shows $\lambda_{c}-\lambda_{p}$ as a function of $L$ in $\log -\log$ scale. The dashed line has slope $1 / v_{\perp}$ as a guide to the eye.

demonstrates, once again, the correctness of the finite-size scaling form for the order parameter of the LS method.

\section{Robustness with respect to the coverage fraction threshold $c_{\mathrm{th}}$}

In the results presented above, we have used a fixed value of the coverage fraction threshold $c_{\text {th }}=0.5$. As we have discussed in Sec. IV, our results are, however, independent of the precise value of $c_{\text {th }}$. To check such a claim, we perform additional simulations for threshold values $c_{\text {th }}=0.75$ and $c_{\text {th }}=0.90$. In Fig. 6(a), we plot the average lifespan as a function of $\lambda$ for a fixed system size, $L=5000$, and different values of the coverage fraction threshold $c_{\text {th }}$. As we can see, increasing the coverage fraction threshold slightly shifts both the position of the peak as well as the height of the maximum lifespan. Nevertheless, as we show in Fig. 6(b) the height of the peak of $\langle\tau\rangle$ scales with the system size $L$ with an exponent $(1-\delta) / \mu=1.32$ that is independent of $c_{\text {th }}$.

In Fig. 7, we show the extrapolation of the different values of the position of the peak $\lambda_{p}^{\mathrm{LS}}(L)$, applying Eq. (16). As we can see, all values of $c_{\text {th }}$ lead asymptotically to the same value of $\lambda_{c}$. This fact indicates that the critical properties of the model are recovered in the LS method in a robust way, independently of the arbitrary choice of the coverage fraction threshold $c_{\text {th }}$. In this sense, it is noticeable that, although the $\lambda_{p}^{\mathrm{QS}}(L)$ values obtained via the $\mathrm{QS}$ method approach the critical point faster than those obtained using LS (see Fig. 2), if the value of the coverage fraction threshold $c_{\text {th }}$ in the LS simulation is increased, the convergence to the asymptotic value of the critical point becomes faster in $L$, although computationally more expensive.

\section{CONCLUSIONS}

The precise determination of the critical properties of absorbing-state phase transitions is a crucial problem in nonequilibrium statistical mechanics. Indeed, while powerful analytical strategies, such as field theoretic methods and their 
renormalization group analysis, are available, these methods are technically complex and ensuing loop expansions lead to approximate values for critical exponents, sometimes of uncontrolled validity in physical dimensions. For this reason, good numerical tools are of invaluable help. Here, we have reported a numerical technique, the lifespan method, which is able to determine with great accuracy the critical properties of absorbing-state phase transitions. To this end, we have developed the corresponding finite-size scaling theory, which allows us to determine precisely both the critical point and the critical exponents by looking at the size dependence of the associated susceptibility and order parameter. Results of the application of the lifespan method to the contact process in a $d=1$ lattice are compared with results from the quasistationary method and other numerical and analytical results, showing that the approach is fully reliable. We note that, even though the LS method has been validated here for an absorbing-state phase transition to a unique absorbing state, it can be generalized to systems with many such states.
To sum up, the lifespan method is an alternative way to numerically studying systems with absorbing states, which complements more traditional techniques, such as the quasistationary method, and that will represent in the future a useful addition to the numerical tool set of the statistical physics practitioner.

\section{ACKNOWLEDGMENTS}

R.P.-S. acknowledges financial support from the Spanish MINECO, under Projects No. FIS2010-21781-C02-01 and No. FIS2013-47282-C2-2, EC FET-Proactive Project MULTIPLEX (Grant No. 317532), and ICREA Academia, funded by the Generalitat de Catalunya. R.P.-S. and A.S.M. acknowledge financial support from CAPES under Project No. 5511-13-5. M.B. acknowledges financial support from the James S. McDonnell Foundation; the ICREA Academia foundation, funded by the Generalitat de Catalunya; MINECO Project No. FIS2013-47282-C2-1-P; and Generalitat de Catalunya Grant No. 2014SGR608.
[1] O. Diekmann and J. Heesterbeek, Mathematical Epidemiology of Infectious Diseases: Model Building, Analysis and Interpretation (Wiley, New York, 2000).

[2] M. Henkel, H. Hinrichsen, and S. Lübeck, Non-equilibrium Phase Transition: Absorbing Phase Transitions (SpringerVerlag, Dordrecht, 2008).

[3] J. Marro and R. Dickman, Nonequilibrium Phase Transitions in Lattice Models (Cambridge University Press, Cambridge, UK, 1999).

[4] G. Schütz, in Phase Transitions and Critical Phenomena, edited by C. Domb and J. Lebowitz (Academic Press, New York, 2001), Vol. 19, pp. 1-251.

[5] U. C. Täuber, Critical Dynamics (Cambridge University Press, Cambridge, UK, 2014).

[6] V. Elgart and A. Kamenev, Phys. Rev. E 74, 041101 (2006).

[7] G. Ódor, Phys. Rev. E 51, 6261 (1995).

[8] I. Jensen, J. Phys. A: Math. Gen. 29, 7013 (1996).

[9] I. Jensen, J. Phys. A: Math. Gen. 32, 5233 (1999).

[10] Finite Size Scaling, Current Physics-Sources and Comments, Vol. 2, edited by J. L. Cardy (North-Holland, Amsterdam, 1988).
[11] M. M. de Oliveira and R. Dickman, Phys. Rev. E 71, 016129 (2005).

[12] R. Dickman, Phys. Rev. E 73, 036131 (2006).

[13] R. Dickman and R. Vidigal, J. Phys. A: Math. Gen. 35, 1147 (2002).

[14] R. Pastor-Satorras, C. Castellano, P. V. Mieghem, and A. Vespignani, arXiv:1408.2701.

[15] M. Boguñá, C. Castellano, and R. Pastor-Satorras, Phys. Rev. Lett. 111, 068701 (2013).

[16] T. E. Harris, Ann. Prob. 2, 969 (1974).

[17] D. Gillespie, J. Phys. Chem. 81, 2340 (1977).

[18] D. Gillespie, J. Comput. Phys. 22, 403 (1976).

[19] S. C. Ferreira, C. Castellano, and R. Pastor-Satorras, Phys. Rev. E 86, 041125 (2012).

[20] K. Binder and D. Heermann, Monte Carlo Simulation in Statistical Physics: An Introduction (Springer, New York, 2010).

[21] J. Brankov and N. Tonchev, J. Stat. Phys. 60, 519 (1990).

[22] D. Stauffer and A. Aharony, Introduction to Percolation Theory, 2nd ed. (Taylor \& Francis, London, 1994). 